\title{
Preface
}

\section{Challenges in the Evaluation and Management of Thoracic Outlet Syndrome}

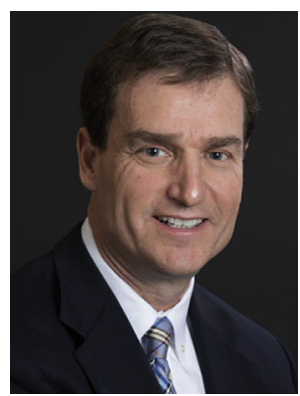

Dean M. Donahue, MD

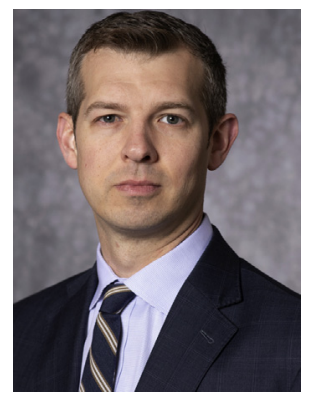

Hugh G. Auchincloss, MD

Editors

Thoracic outlet syndrome (TOS) represents 3 separate, potentially overlapping conditions that will each be discussed in this issue. The vascular forms of this condition (venous and arterial TOS) have more established diagnostic and management criteria. Treating these conditions still requires careful evaluation and skillful surgical management, and these are outlined in 2 outstanding articles in this issue.

Neurogenic TOS is among the most challenging and controversial conditions that a clinician can face. While there have been recent attempts to address this deficiency, there are still is no established consensus diagnostic and therapeutic criteria. Treatment algorithms may vary widely among clinicians, and randomized controlled trials are lacking. Currently, clinicians must rely on their experience and intuition to formulate a treatment pathway for each individual patient. With more exposure to TOS patients and careful observation, clinicians may be able to identify patterns that allow them to more accurately predict the probability of a given treatment outcome. This requires spending time with the patient and carefully listening to and documenting their symptoms.

Ideally, as we develop a common language regarding the evaluation of TOS, a more precise diagnosis and potential rules-based therapeutic strategy may be possible. The goal of this issue, and other recently published textbooks on this condition, is to continue on the path to developing this common language.
We are indebted to $\mathrm{Dr}$ M. Blair Marshall, the former Consulting Editor of Thoracic Surgery Clinics, for the opportunity to assemble a collection of insightful contributions provided by several outstanding clinicians with expertise in TOS. We would also like to thank Laura Fisher and John Vassallo for their invaluable assistance in bringing this issue to fruition. We are also deeply indebted to the dedicated team of the Massachusetts General Hospital Thoracic Outlet Syndrome Program: Julie Donahue, RN, Kathy Jackson, NP, Lauren Taylor, RN, and A. Daniel Muzorewa.

Dean M. Donahue, MD Department of Thoracic Surgery Massachusetts General Hospital

Founders 734

55 Fruit Street

Boston, MA 02114, USA

Hugh G. Auchincloss, MD Department of Thoracic Surgery Massachusetts General Hospital

Founders 742

55 Fruit Street

Boston, MA 02114, USA

E-mail addresses:

ddonahue@mgh.harvard.edu (D.M. Donahue) hauchincloss@mgh.harvard.edu

(H.G. Auchincloss) 